\title{
Apercepción y conscientia en la ontología monadológica de Leibniz
}

\author{
ROBERTO CASALES GARCÍA* \\ Universidad Popular Autónoma del Estado de Puebla (México) \\ roberto.casales@upaep.mx
}

\begin{abstract}
Resumen
El objetivo principal de este artículo es analizar la distinción leibniziana entre apercepción sensible y conscientia a la luz de su ontología monadológica, con la intención de esclarecer las diferencias constitutivas entre los tres tipos de mónadas que Leibniz postula, esto es, entre las mónadas simples, las meras almas y los espíritus. Con esto, además de argumentar en contra de la concepción estándar de la apercepción, la cual termina por confinarla al caso específico de los espíritus, sitúo la propuesta del hannoveriano entre dos lecturas contemporáneas: por un lado, aquella que comprende la apercepción como un acto de orden superior reflexivo, esto es, una percepción de la percepción; y, por otro lado, aquella que, al distinguir entre apercepción sensible y conscientia, privilegia una teoría de primer orden para hablar de la primera, reservando los actos reflexivos para la segunda. Aunque la evidencia textual es más afín con la segunda, hay elementos de la primera que permiten matizarla.
\end{abstract}

Palabras claves: percepción, apetito, apercepción/conciencia, sensación, Leibniz.

\section{Apperception and conscientia in Leibniz's monadological ontology}

\begin{abstract}
The main aim of this article is to analyze Leibniz's distinction between sensitive apperception and 'conscientia' through his monadological ontology, with the intention of lighten the constitutive differences between the three types of monads that Leibniz state, that is, between bare monads, souls and spirits. By proving this, my approach not only argues against the standard conceptions of Leibniz's notion of apperception, which ends attributing apperception only to the specific case of spirits, but it also places the Hanoverian proposal between two contemporary lectures: on one side, an approach that understand apperception as a high order reflexive act, that is, a perception of a perception; on the other side, an approach that distinguishes sensible apperception from 'conscientia' in order to privilege a first order theory for the first one, reserving the reflective acts only for the second. Even when the textual evidence is closer to the last one, there are some elements of the first approach that allow us to improve it.
\end{abstract}

Keywords: Perception, appetite, apperception/consciousness, sensation, Leibniz:

* Doctor en Filosofía por la Universidad Nacional Autónoma del Estado de México. Profesor investigador y director académico de la Facultad de Filosofía de la UPAEP. Es autor de Imperativo categórico y carácter. Una introducción a la filosofía práctica de Kant (2019), Justicia, amor e identidad en la ontología monadológica de Leibniz. (2018), editor de Leibniz en México. Homenaje a Alejandro Herrera (2018), La modernidad en perspectiva. A trescientos años del fallecimiento de Leibniz (2017), La esencia del amor (2017), y Libertad y necesidad en Leibniz: Ensayos sobre el laberinto leibniziano de la libertad (2015), entre otras publicaciones.

Recibido: 15/Noviembre/2018 - Aceptado: 4/Marzo/2019 


\section{INTRODUCCIÓN}

Una de las primeras distinciones entre la concepción cartesiana de la mente y aquella que formula Leibniz, radica en que el primero, además de comprender los actos mentales bajo el concepto de pensamiento, sostiene que todo acto mental de las sustancias incorpóreas es consciente (Descartes, 1982-91: VIII, 160), mientras que el hannoveriano, al entender estos actos en términos de percepción y apetición, admite la existencia de actos mentales carentes de apercepción o conciencia. Tal es el caso de las pequeñas percepciones, de las cuales no damos cuenta porque sus "impresiones son o demasiado pequeñas a la par que excesivas en número, o están demasiado juntas, de manera que no tienen nada que permita distinguirlas por separado, pero aunque estén unidas a las otras no por ello dejan de producir efecto y de hacerse notar en el conjunto, aunque sea confusamente" (Leibniz, 1992: 42 [GP, V, 46-47]) ${ }^{1}$. Con esto, además de distinguir entre actos mentales conscientes e inconscientes, se admite también que la apercepción admite cierta limitación, como ocurre en el caso de la apercepción sensible, la cual, al conformarse por una infinidad de pequeñas percepciones, encierra cierta confusión inherente que le impide distinguir cada una de éstas con claridad ${ }^{2}$.

La apercepción sensible, en este sentido, se concibe como un tipo de conciencia fenoménica que nos permite dar cuenta, al menos de forma confusa, de aquellas impresiones recibidas a través de los órganos sensoriales. Cuando hablamos de la sensibilidad en Leibniz, sin embargo, observamos una cierta distinción entre aquellas percepciones sensibles que apercibimos y aquellas de las cuales no reparamos, tal y como ocurre cuando

1 Todas las referencias a la obra de Leibniz se citan primero acorde a la edición en castellano citada, y después, entre corchetes, acorde a la edición original del texto, siguiendo las abreviaturas canónicas del autor (ver Leibniz, 1923ss., 1948, 1954, 1961, 1965, 1997). En el caso de aquellas citas que no cuentan con una edición en castellano y cuya traducción es propia, se cita sólo la edición del texto original.

2 Esto se puede apreciar con mayor claridad en dos pasajes significativos: en primer lugar, en el SXXXIII de su Discours de métaphysique, donde sostiene que "las percepciones de nuestros sentidos, incluso cuando son claras, deben necesariamente contener algún sentimiento confuso, pues como todos los cuerpos del universo están en sintonía, el nuestro recibe la impresión de todos los demás, y aunque nuestros sentidos se refieran a todo, no es posible que nuestra alma pueda atender a todo en particular; ese es el motivo por el que nuestros sentimientos confusos son el resultado de una variedad de percepciones, que es totalmente infinita" (Leibniz, 2010: 199 [AA VI, 4B, 1582-1583]); en segundo lugar, en su De formis seu attributis Dei, donde señala que "las cosas sensibles no pueden ser entendidas perfectamente por nosotros, porque para su constitución concurren cosas infinitas, a causa de la divisibilidad al infinito del tiempo y el lugar. En virtud de esto, sucede que la percepción de la cualidad sensible no es una percepción, sino un agregado de infinitas percepciones" (Leibniz, 2010: 81 [AA VI, 3, 515]). 
dormimos, ya que "mientras se duerme, nunca deja de tenerse algún sentimiento, más o menos débil, incluso cuando no se sueña" (Leibniz, 1992: 119 [GP, V, 104]). Lo cual nos permite distinguir, a su vez, entre dos conceptos distintos de sensación, en cuanto que, así como hay percepciones simples carentes de apercepción, encontramos percepciones que se elevan al grado de la sensación sin por eso ser apercibidas. De modo que, a pesar de que el hannoveriano a menudo alude a la sensación teniendo en mente los actos conscientes, como se puede apreciar en la evidencia textual que ofrece Kulstad (1981: 39-42) al respecto, observamos ciertas sensaciones que, al no estar acompañadas de memoria, no son susceptibles de ser apercibidas (Barth, 2014: 338).

Aunque, en sentido estricto, la apercepción se presenta hasta el nivel de la sensación, particularmente en aquellas sensaciones que van acompañadas de memoria, existe evidencia textual que nos permite distinguir entre la apercepción sensible y el tipo de apercepción que se da a través de los actos reflexivos, esto es, entre la conciencia fenoménica y la conscientia, la cual alude no tanto a la capacidad de dar cuenta de los objetos externos y de nuestras impresiones sensibles, como a la capacidad del sujeto de apercibirse a sí mismo y a sus operaciones internas. Distinción que se hace presente de manera más precisa cuando el hannoveriano, en sus Nouveaux essais sur l'entendement humain, distingue entre apercibir y entender:

Nos damos cuenta de muchas cosas en nosotros y fuera de nosotros que en principio no entendemos; pero cuando llegamos a tener ideas distintas, mediante el poder de reflexionar y de deducir verdades necesarias, entonces las entendemos. En este sentido las bestias no tienen entendimiento, aunque posean la facultad de apercibirse de las impresiones más destacables y distinguidas, al modo en que el jabalí advierte a una persona que le grita, y va derecho hacia dicha persona, aunque hasta entonces no había tenido de ella más que una percepción pura, pero confusa, como de todos los demás objetos que caían en su campo visual y cuyos rayos hieren su cristalino. Según mi modo de entenderlo, el entendimiento se corresponde con lo que los latinos llamaban intellectus, y el ejercicio de dicha facultad se llama intelección, y consiste en una percepción distinta, junto con la facultad de reflexionar, que no existe en los animales. (Leibniz, 1992: 197-198 [GP, V, 159])

El problema se presenta cuando confrontamos esta serie de distinciones con algunas concepciones estándar de la apercepción, ya que éstas tienden a vincular la apercepción con los actos reflexivos ${ }^{3}$, atribuyendo a

Pienso de forma concrete en de la lectura de Robert McRae (1978: 32), quien sostiene que la apercepción es una capacidad o atributo exclusivo de los espíritus, en cuanto sólo éstos poseen actos reflexivos. Quizá una lectura estándar de la apercepción más 
Leibniz una posición inconsistente (McRae, 1978: 34). La inconsistencia se presenta entre el pasaje citado, donde el hannoveriano atribuye a los animales apercepción más no reflexividad, y la definición de apercepción que aparece en el $\$ 4$ de sus Principes de la nature et de la grâce fondés en raison, en el que concibe la apercepción en términos de conocimiento reflexivo (Leibniz, 2010: 354 [Robinet I, 37]). Aunado a esta inconsistencia, encontramos dos aproximaciones contemporáneas al tema de la apercepción en Leibniz, a saber: por un lado, las que conciben la apercepción como una operación reflexiva de segundo grado, esto es, una percepción de la percepción; por otro, aquellas que distinguen entre apercepción sensible y conscientia, articulando una teoría de primer orden para la conciencia fenoménica y reservando la reflexividad para la segunda. Así, mientras la primera aproximación considera la apercepción como un acto de orden superior (bigher-order theory), esto es, que una percepción es apercibida en virtud de un segundo acto intencionalmente dirigido a ese estado mental (Jorgensen, 2009: 236); las aproximaciones de primer orden (first-order account) sostienen que la apercepción sensible o conciencia fenoménica depende de ciertas características o condiciones propias del estado mental en cuestión (Barth, 2014: 334).

Mi propuesta, tal y como pretendo evidenciar a lo largo de este trabajo de investigación, consiste en defender que Leibniz, al distinguir entre apercepción sensible y conscientia, se sitúa en un punto intermedio entre estas dos aproximaciones, lo cual es fundamental para entender la relación entre los niveles o grados de percepción que propone (percepción simple, sensación e intelección) y los tipos de mónadas que conforman su ontología monadológica (simples mónadas, almas y espíritus). Para lograr este objetivo realizaré tres cosas: en primer lugar, analizo en qué sentido afirma Leibniz que percepción y apetición son los principios internos del cambio, y en qué medida estos explican la actividad mental de las mónadas; en segundo lugar, reconstruyo una concepción leibniziana de la apercepción sensible o conciencia fenoménica a partir de la relación entre distinción, memoria y atención; finalmente, en tercer lugar, articulo una concepción de la conscientia desde la reflexividad, lo cual, si mi hipótesis es cierta, nos permite esclarecer los tipos de mónadas que el hannoveriano distingue en sus obras de madurez.

radical sea la que Nicholas Rescher, en cuanto que éste, al vincular la apercepción con la reflexión, comprende la apercepción en términos de autoconciencia y no de conciencia fenoménica o de apercepción sensible (Rescher, 1979: 119-120). Cabe matizarse, sin embargo, que Rescher, al entender la apercepción en términos de autoconciencia, no niega que existan en Leibniz sensaciones conscientes, lo cual, de alguna, permite salvaguardar la inconsistencia respecto a la conciencia animal (1979: 120). 


\section{PERCEPCIÓN Y APETICIÓN COMO PRINCIPIOS INTERNOS DEL CAMBIO}

La teoría leibniziana de la percepción y la apercepción (en general), tal y como se puede apreciar en su Monadologie (Leibniz, 2010: 328-332 [GP, VI, 608-612]), se inserta en el marco de su ontología monadológica y de su comprensión dinámica de la realidad, según la cual "todo ser creado está sujeto a cambio y, en consecuencia, también la mónada creada, e incluso que dicho cambio es continuo en cada una" (Leibniz, 2010: 329 [GP, VI, 608]). Para Leibniz, en efecto, 'substancia' y 'acción' son dos caras de una misma moneda, en cuanto que "por su propia naturaleza una sustancia no puede existir sin acción” (Leibniz, 1992: 41 [GP, V, 46]; véase: Leibniz, 2010: 344 [Robinet I, 27]) y, en consecuencia, "no se sigue que entonces la sustancia simple carezca de toda percepción" (Leibniz, 2010: 330 [GP, VI, 610]; véase también: Leibniz, 1992: 115-116 [GP, V, 101]). De ahí que Leibniz conciba las mónadas como unidades sustanciales primitivas que, al no estar desprovistas de percepción y apetitos, tienden en todo momento a la acción, donde cada una constituye un "sujeto primitivo de la vida y de la acción, dotada siempre de percepción y de apetición, que encierra en sí siempre, junto con lo que es, la tendencia a lo que será [en consecuencia siempre subsistente] para representar cualquier otra cosa que exista en el futuro" (Leibniz, 2010: 275 [Grua, 554]).

Las mónadas leibnizianas, en este sentido, constituyen unidades substanciales cuyo dinamismo interno radica en su naturaleza perceptual y apetitiva, en cuanto la percepción constituye el detalle de lo que cambia, y el apetito la acción del principio interno por la cual se realiza el paso de una percepción a otra (Leibniz, 2010: 329 [GP, VI, 608-609]). Según esta última caracterización, la percepción es tanto el "estado transitorio que envuelve y representa una multitud en la unidad" (Leibniz, 2010: 329 [GP, VI, 608]), como "la representación de lo externo en lo interno" (Rovira, 2006: 78 [GP, VII, 529]); mientras que por apetito se entiende prioritariamente la tendencia de una percepción a otra (Rovira, 2006: 35 [GP, III, 575]). Dado esto último, es decir, que las mónadas sólo pueden transitar de una percepción a otra en virtud de sus apetitos o tendencias, se sigue que la apetición es lo que da cohesión y unidad a cada una las mónadas, garantizando con ello la 'armonía del percipiente' (Leibniz, 2011b: 1225 [GP, II, 270]). A partir de lo cual se siguen dos cosas: por un lado, que no hay percepción sin apetición; por otro lado, que la apetición juega un papel central en la constitución de las mónadas, particularmente en lo que compete a su individualidad, ya que, como se aprecia en el $\$ 291$ de la tercera parte de sus Essais de Theodicée, es la ley de los apetitos la que garantiza el enlace sistemático de la serie de percepciones que entran dentro de la noción completa de cada sustancia (Leibniz, 2012: 297 [GP, VI, 289]). 
Al concebir la vida interna de las mónadas en términos de percepción y apetición, y sostener que no hay percepción sin apetición, se afirma también que el apetito juega un papel central en la génesis misma de las percepciones, tesis fundamental para comprender la noción leibniziana de espontaneidad. En efecto, dado que "todo lo que acontece en el alma y en cada sustancia es algo que se sigue de su noción", de modo que "todas sus apariencias o percepciones deban surgirle (sponte) de su propia naturaleza" (Leibniz, 2010: 199 [AA VI, 4B, 1582]), y que el apetito es esa fuerza o conato que posibilita la tendencia de una percepción a otra ${ }^{4}$, se sigue que todas "las percepciones en la mónada nacen unas de otras según las leyes de los apetitos o de las causas finales del bien y del mal, que consisten en las percepciones notables, regulares o irregulares" (Leibniz, 2010: 345 [Robinet I, 31-33]). A lo cual se añade que el apetito, a pesar de que "no siempre puede alcanzar completamente toda percepción a la que tiende", "siempre obtiene algo de ella y llega a percepciones nuevas" (Leibniz, 2010: 329 [GP, VI, 609]), sin lo cual habría discontinuidad al interior mismo de las mónadas. Para Leibniz, en resumen, la carencia de apetitos implicaría la imposibilidad de transitar de una percepción a otra y, por tanto, la carencia de vida interna de las mónadas, cosa equivalente a su aniquilación.

De ahí que la apetición juegue un papel central dentro de la teoría leibniziana de la percepción y que, por tanto, sea indispensable para comprender tanto los distintos grados de percepción que el hannoveriano postula -percepción simple, sensación y pensamiento (Barth, 2014: 337)-, como su consecuente taxonomía de las mónadas -mónadas simples, almas y espíritus (Herrera, 1993: 98-99). En efecto, puesto que en Leibniz no hay percepción sin apetición, en cuanto que es indispensable para transitar de una percepción a otra, se sigue que toda mónada posee apetitos ${ }^{5}$ (Leibniz, 2010: 330 [GP, VI, 610]), sin importar si la totalidad de sus percepciones son confusas, como ocurre con las mónadas simples y su estado de perpetuo aturdimiento (Leibniz, 2010: 331 [GP, VI, 611]). Para estas últimas,

$4 \quad$ Acorde a la lectura de Rutherford (2005: 162), decir que las mónadas son espontáneas significa que éstas poseen la fuerza suficiente para determinar cada uno de sus estados sucesivos, de modo que todas las sustancias simples, como se aprecia en el $\$ 18$ de su Monadologie, "tienen en sí mismas cierta perfección ( $(\alpha \dot{U} \tau \alpha \varrho \chi \varepsilon i \alpha)$ que las convierte en fuente de sus acciones internas y, por así decirlo, en autómatas incorpóreos" (Leibniz, 2010: 330 [GP, VI, 609-610]).

5 Algo semejante se aprecia en la caracterización de las mónadas como fuerzas primitivas, tal y como se aprecia en su correspondencia con de Volder, donde sostiene que "las fuerzas primitivas no pueden ser otra cosa que las tendencias internas de las sustancias simples, mediante las cuales éstas pasan de percepción a percepción en virtud de una determinada ley de su naturaleza" (Leibniz, 2011b: 1231 [GP II, 275]). A partir de lo cual se sigue, tal y como observa Andreu, percepción y apetito "son una misma cosa en movimiento" (2015: XX). 
dado que la totalidad de sus percepciones son confusas, se sigue que sus apetitos o tendencias son en cierto modo pasivas o ciegas, caracterización que concuerda con el $\$ 49$ de su Monadologie, en cuanto que en este pasaje atribuye "acción a la mónada en tanto que tiene percepciones distintas, y pasión en tanto que las tiene confusas" (Leibniz, 2010: 335 [GP, VI, 615]). Este tipo de apetitos, sin embargo, no son exclusivos de las mónadas simples, ya que en las almas y los espíritus también experimentan casos de aturdimiento momentáneo, como ocurre con la muerte o cuando dormimos (Leibniz, 2010: 346 [Robinet I, 37]; véase también: Leibniz, 2010: 330 [GP, VI, 610]).

La vida interna de las mónadas, según lo anterior, mantiene una estructura compleja, la cual oscila entre aquella infinitud de pequeñas percepciones y sus correspondientes apetitos ciegos, y los grados más altos de percepción y apetición, que sólo poseen los espíritus, esto es, el entendimiento y la voluntad. Incluso en el caso de las almas y de los espíritus, cuyas percepciones no siempre son confusas como en las mónadas simples, encontramos diversos grados de complejidad y de perfección en sus percepciones y apetitos, de modo que la actividad mental de cada individuo varía en todo momento. La existencia de estas variaciones internas en la naturaleza perceptual y apetitiva de cada mónada, particularmente de las almas y los espíritus, es fundamental para entender por qué, a pesar de que siempre están percibiendo, no siempre logran dar cuenta de todas sus percepciones. Más aún, si mi hipótesis es correcta, la existencia de distintos grados de percepción y apetición en las mónadas nos permite comprender mejor la distinción entre percepción simple, apercepción sensible y conscientia, sin necesidad de hablar de percepciones de segundo orden, ni de excluir la conciencia sólo a los espíritus.

\section{APERCEPCIÓN SENSIBLE O CONCIENCIA FENOMÉNICA}

Si la percepción constituye el detalle de lo que cambia y el apetito la acción del principio interno que explica el paso o tendencia de una percepción a otra, tal y como acabo de mencionar, entonces es posible concluir que el acto de percibir involucra ambos elementos, de modo que no es necesario entender la percepción y la apetición como actos distintos, sino tan sólo como dos elementos constitutivos de un mismo acto. Así, el primer problema de las lecturas estándar de la apercepción sensible en Leibniz, radica en olvidar esta singularidad de la teoría leibniziana de la percepción, para centrarse de forma exclusiva en la naturaleza expresiva de las mónadas. De ahí se desprende también un segundo problema, a saber, que estas lecturas, al no concebir la percepción y la apetición como un mismo acto, se ven en la necesidad de acudir a las teorías de orden 
superior para explicar la conciencia, la cual terminan por caracterizar como una percepción de la percepción: una percepción de primer orden de $\mathrm{X}$ y una percepción reflexiva de segundo orden sobre la percepción original de X (Simmons, 2011: 53; véase también: Genaro, 1999: 353ss) ${ }^{6}$. Esta caracterización de la apercepción como un acto reflexivo de segundo orden, sin embargo, es objetable en cuanto que deja sin resolver la inconsistencia relativa a la apercepción animal (Barth, 2011a: 38-39).

La presencia de esta inconsistencia, a su vez, nos permite vislumbrar un tercer problema, a saber, que las lecturas estándar carecen de una distinción clara entre la apercepción sensible o conciencia fenoménica y la conscientia, cuyos rasgos constitutivos son la reflexión y la introspección. Así, mientras la primera se limita reparar en aquellas sensaciones que, por su grado de distinción, son más destacadas y, por tanto, más susceptibles de ser notadas - perceptions notables- (Leibniz, 1992: 116-117 [GP, V, 102103]), la segunda nos remite a aquella capacidad reflexiva de dar cuenta de su propio ser y su obrar, que sirve de base para constituir la propia agencia moral y, por ende, la identidad personal (Leibniz, 2010: 200 [AA VI, 4B, 1583-1584]). Las lecturas estándar de Leibniz, en este sentido, tienden a unificar ambos aspectos de la conciencia bajo la noción de apercepción, explicando ambos a través de la reflexividad, lo cual, además de hacer inconsistente su taxonomía de las mónadas, termina por obnubilar el papel de la reflexión ${ }^{7}$. De ahí que sea necesario elaborar una lectura más robusta sobre la conciencia en Leibniz, mediante la cual se ofrezca una posible solución a estos tres problemas.

Uno de los elementos clave para elaborar una lectura de este tipo, a mi parecer, se aprecia en el $\$ 19$ de su Monadologie, en donde el hannoveriano distingue entre las meras entelequias y las almas en función de sus

6 Cabe señalarse, sin embargo, que la expresión 'percepción de la percepción' no es del todo ajena al filósofo de Hannover, tal y como se puede ver en un pasaje de su $D e$ natura mentis et corporis en el que afirma que "una cosa es percibir, otra cosa es percibir que uno percibe, o recordar" (AA VI, 4B, 1490: la traducción es propia). Algo semejante ocurre en la definición de memoria que aparece en sus Definitiones cogitationesque metaphysicae, según la cual "la memoria es la percepción de las propias percepciones" (AA VI, 4B, 1394: la traducción es mía). La ambigüedad que generan estos pasajes, sin embargo, se puede resolver si consideramos el papel de la apetición en la teoría leibniziana de la percepción y si consideramos elaboramos una teoría más robusta de la memoria en Leibniz, tal y como sugiere Jorgensen (2011: 888), con la salvedad de conservar ambas en el contexto de una teoría de primer orden, como pretendo mostrar en este apartado.

$7 \mathrm{Al}$ distinguir entre reflexión simple y reflexión lockeana, Kulstad ofrece una alternativa viable para mantener la comprensión reflexiva de la conciencia (Kulstad, 1981: 3334). Esta alternativa, a pesar de que resuelve algunas de las inconsistencias internas de las lecturas estándar, carece de evidencia textual suficiente (Barth, 2011a: 39), además de ser susceptible de los primeros dos problemas que acabo de mencionar. 
distintos grados de percepción: dado que el sentimiento es más que una simple percepción, en palabras de Leibniz, "concedo que baste el nombre general de mónadas y de entelequias para las sustancias simples que sólo gocen de eso, y que se llamen almas solamente a aquellas cuya percepción es más distinta y va acompañada de memoria" (Leibniz, 2010: 330 [GP VI, 610]). Acorde con este pasaje, mientras las meras entelequias se limitan a poseer simples percepciones y apetitos ciegos, las almas son capaces de sensación, lo cual implica dos cosas: por un lado, que éstas poseen percepciones con un mayor grado de distinción o relieve; y, por otro lado, que éstas van acompañadas de memoria, de modo que un eco de lo percibido perdura durante largo tiempo "para dejarse oír ocasionalmente" (Leibniz, 2010: 345 [Robinet I, 33-35]). Distinción y memoria, por tanto, se presentan como elementos clave tanto para entender la noción leibniziana sensación, en sus dos acepciones, como para reconstruir su concepción de la apercepción sensible.

Que una percepción sea más distinta que otra, en primer lugar, significa que ésta posee cierto relieve o cualidad que la hace suficientemente notable como para ser captada ${ }^{8}$ (Leibniz, 1992: 222 [GP, V, 180]; léase en conjunto con: Leibniz, 2010: 331 [GP, VI, 611]). Una idea o una percepción es clara, acorde con la propuesta de Leibniz, cuando ésta es suficiente para reconocer y distinguir una cosa de otra (Leibniz, 1992: 291-292 [GP, $\mathrm{V}, 236]$ ); pero es distinta cuando, además de esto, podemos discernir las notas que entran dentro de su definición, de modo que una misma sensación puede ser clara y confusa al mismo tiempo: "son claras, pues se las reconoce y discierne fácilmente unas de otras, pero no son distintas, porque no se distingue lo que contienen" (Leibniz, 1992: 292 [GP, V, 237]). Esto mismo ocurre con todas nuestras sensaciones, las cuales, al componerse por una infinidad de pequeñas percepciones, son claras en cuanto componen un todo, pero confusas en la medida en que no podemos distinguir perfectamente cada una de esas pequeñas percepciones (Leibniz, 2010: 199 [AA VI, 4B, 1582-1583]; véase también: Leibniz, 2010: 81 [AA VI, 3, 515]).

Para comprender mejor esto último, sin embargo, es necesario señalar que las sensaciones, a diferencia de las percepciones simples, "son representaciones de cierto tipo de estados corpóreos, a saber, estados de los órganos sensoriales" (Barth, 2014: 338), es decir, representaciones de las

8 A este respecto, conviene señalar cómo se distingue esto último de las teorías de orden superior, ya que en éstas se defiende también la idea de que los grados de distinción tiene que ver con la cualidad de las mismas de ser destacadas o notables. La diferencia fundamental radica en que para estas teorías, ser captable significa que son susceptibles de ser notadas por un acto de segundo orden (Simmons, 2011: 57-58), mientras que en esta lectura se sostiene que este segundo acto no es necesario a este nivel. 
impresiones sensibles que captamos mediante nuestros órganos sensoriales, tal y como se aprecia en el $\$ 4$ de sus Principes de la nature et de la grâce fondés en raison: "pero cuando la mónada tiene órganos tan ajustados que mediante ellos hay relieve y distinción en las impresiones que reciben y, por consiguiente, en las percepciones que las representan (como, por ejemplo, cuando, mediante la figura de los humores de los ojos, se concentran los rayos de la luz y actúan con más fuerza, puede llegarse hasta el sentimiento..." (Leibniz, 2010: 345 [Robinet I, 33]). Esto significa que el relieve o cualidad en virtud del cual una percepción es más distinta que otra, por ende, se relaciona con el relieve y distinción de las impresiones que reciben nuestros órganos sensoriales, de forma que los grados de distinción de nuestras percepciones aluden a la fuerza de las impresiones sensibles que captan estos órganos (Barth, 2014: 339).

Dada la correspondencia que el hannoveriano postula entre el alma y el cuerpo (Leibniz, 2010: 248 [GP, IV, 485]), se sigue también que hay una correspondencia entre la fuerza con la que nuestros órganos sensoriales reciben tal o cual impresión, y el grado de distinción de su correspondiente representación mental. Esto ocurre, por ejemplo, con el ojo, el cual, al reunir "varios rayos de luz o muchas ondulaciones del aire para que por esa reunión sean más eficaces" (Leibniz, 2010: 331 [GP, VI, 611]), logra una obtener una impresión mucho más fuerte y, consecuentemente, una sensación con mayor fuerza mental ${ }^{9}$. Que una sensación goce una mayor fuerza mental y, por tanto, de un mayor grado de distinción, sin embargo, no es condición suficiente para hablar de apercepción sensible, como sostiene Jorgensen (2009: 242) en un primer momento. A pesar de que el grado de distinción es suficiente para transitar de la mera percepción al sentimiento, como sostiene en su Specimen inventorum admirandis naturae generalis arcanis de 1688 (AA, VI, 4B, 1625: "quodsi perceptio sit distinctior, sensum facit"), esta noción de sentimiento alude prioritariamente a aquellas sensaciones que percibimos sin por eso apercibirlas, como ocurre mientras dormimos (Barth: 2014: 342).

Acorde con la propuesta del hannoveriano, lo característico de este primer tipo de sensaciones radica en que poseen un mayor grado de distinción, esto es, de fuerza mental y/o relieve, más no el suficiente para ser acompañadas por la memoria y, por tanto, para ser apercibidas. De ahí que sea necesario, en segundo lugar, hablar de la memoria con una condición

$9 \quad$ En el caso específico de la sensación, conviene recordar que ésta, a pesar de que se presenta como una sola percepción, sino, en palabras de Leibniz, "un agregado de infinitas percepciones" (Leibniz, 2010: 81 [AA, VI, 3, 515]), las cuales, si mi lectura es correcta, son agrupadas mediante el apetito para lograr una mayor fuerza mental. 
necesaria que toda sensación debe satisfacer para ser apercibida (Jorgensen, 2011: 888). Para entender el papel que juega la memoria en la apercepción sensible, si mi lectura es correcta, es necesario discernir a qué noción de memoria se refiere Leibniz cuando habla de la sensación, ya que a lo largo de su propuesta el hannoveriano distingue tres formas o tipos de memoria: la memoria virtual u ontológica, en virtud de la cual toda mónada "contiene siempre trazos de lo que siempre ha sido y marcas de lo que siempre será" (Leibniz, 2007: 37 [Finster, 84]; véase también: Leibniz, 2007: 62; [Finster, 150]); la reminiscencia, cuya función consiste en "reproducir" aquellas sensaciones previas (Leibniz, 1992: 74 [GP, V, 73]) sin que el objeto vuelva a estar presente (Leibniz, 1992: 183 [GP, V, 147]); y el recuerdo (souvenir), a través del cual el sujeto no sólo es consciente de la existencia del objeto externo, sino, además, de sí mismo (Leibniz, 2010: 200 [AA, VI, 4B, 1584]).

A pesar de que el primer tipo de memoria, esto es, la memoria virtual u ontológica, garantiza tanto la continuidad en el flujo de percepciones que posee cada mónada, como una cierta identidad ontológica, de modo que incluso es posible para las substancias racionales revivir algunas de esas percepciones virtuales, como se aprecia en su correspondencia con Arnauld (Leibniz, 2007: 62 [Finster, 150]), queda claro que ésta no es la noción de memoria a la que el hannoveriano hace referencia cuando habla de sensación, en cuanto que ésta alude a toda mónada en general, mientras que la sensación es propia sólo de las almas. Queda claro también que tampoco alude al tercer tipo de memoria, puesto que ésta alude a un tipo de memoria reflexiva que, en consecuencia, sólo es posible encontrar en las substancias racionales. De ahí que la apercepción sensible o conciencia fenoménica se relacione prioritariamente con la reminiscencia, y sólo tangencialmente, en el caso de los espíritus, con el recuerdo (somvenir), lo cual nos permite salvaguardar la aparente inconsistencia que se presenta al hablar de la conciencia en los animales no-humanos, sin la necesidad de aludir a una teoría de orden superior. La reminiscencia, en efecto, no requiere ni de la reflexión ni del conocimiento de sí mismo, sino tan sólo la repetición de la sensación "con su contenido intencional original, sin que el objeto intencional esté presente" (Jorgensen, 2011: 897).

Esto significa que, para hablar de la apercepción sensible o conciencia fenoménica en Leibniz, no basta con decir que las percepciones son almacenadas en la memoria, para lo cual no es necesario que éstas posean un mayor grado de distinción, así como tampoco es necesario un acto reflexivo, ya que, como menciona en sus Considerations sur les Principes de Vie, et sur les Natures Plastiques, par l'Auteur du Systeme de l'Harmonie preétabile, "hay mucha distancia entre el sentimiento, que es común a estas almas, y la 
reflexión, que acompaña a la razón, puesto que nosotros tenemos mil sentimientos sin reflexionar acerca de ellos" (Leibniz, 2011a: 502 [GP, VI, 543]). Para que una sensación sea apercibida, si mi lectura es correcta, es necesario que ésta posea fuerza mental suficiente para producir "de golpe el efecto de un bábito prolongado o de muchas percepciones medianas reiteradas" (Leibniz, 2010: 331 [GP, VI, 611]), de modo que el alma pueda reparar en su contenido. La reminiscencia, en este sentido, "proporciona a las almas una especie de consecución que imita a la razón" (Leibniz, 2010: 331 [GP, VI, 611]), en la medida en que permite establecer una conexión entre los fenómenos percibidos más destacados, tal y como se observa en el $\$ 65$ de sus Essais de Theodicée (Leibniz, 2012: 80-81 [GP, VI, 87]; véase también: Leibniz, 2010: 346 [Robinet I, 39]).

Para que esto último ocurra, esto es, que el alma repare en el contenido de sus sensaciones, es necesario añadir un tercer elemento constitutivo de la sensación, el cual haga justicia a la teoría leibniziana de la percepción, en especial al apetito. Este tercer elemento aparece en una tercera caracterización de la sensación de 1710, según la cual "la sensación es la percepción que encierra algo de distinto y va unida con la atención y la memoria" (Leibniz, 2014a: 135 [GP, VII, 330]). Acorde con este pasaje, para hablar de sensación y apercepción sensible es necesario que el sujeto percipiente posea, además de percepciones distintas y de la reminiscencia, la capacidad de atender a algunas de sus sensaciones, "pues", en palabras de Leibniz, "siempre hay objetos que llaman la atención de nuestra vista o de nuestro oído, y por lo tanto afectan también a nuestra alma, sin que nos demos cuenta, porque nuestra atención está absorta en otros objetos, y ello es así hasta que el objeto sea capaz de atraerse su atención, redoblando su actividad o por cualquier otro medio" (Leibniz, 1992: 119 [GP, V, 105]). Una sensación es apercibida, en este sentido, cuando posee la fuerza o distinción suficiente para producir una disposición habitual novedosa ${ }^{10}$, capaz de ser reproducida por la memoria y, en consecuencia, atraer nuestra atención.

De acuerdo a la Table de definitions que el hannoveriano elabora entre 1702 y 1704, se llama atención a aquel estado mental o pensamiento (co-

10 El énfasis que se hace sobre la novedad de estas disposiciones habituales se debe, entre otras cosas, al fenómeno de la habituación perceptual que ocurre cuando una misma sensación es tan recurrente que deja de distinguirse y, por tanto, de destacarse entre otras sensaciones (Simmons, 2011: 59). Leibniz menciona, a este respecto, dos ejemplos que resultan paradigmáticos: por un lado, las sensaciones generadas por algunos movimientos de nuestro cuerpo, como lo es el de los órganos internos (Leibniz, 2014b: 17 [Grua, 558]); por otro lado, "al movimiento de un molino o de un salto de agua cuando desde hace algún tiempo hemos vivido al lado" (Leibniz, 1992: 42 [GP, V, 47]). 
gitatio) que implica un cierto deseo de conocer (Leibniz, 2014c: 69 [Couturat, 493]). Una caracterización semejante de la atención se aprecia en sus Nouveaux essais sur l'entendement humain, donde afirma que "tenemos atención de aquellos objetos que distinguimos y preferimos respecto a los demás" (Leibniz, 1992: 183 [GP, V, 147]). Cabe aclararse que, aun cuando ambas definiciones aluden a la atención como un tipo de deseo o preferencia, lo cual presupone una atención selectiva de carácter voluntario, a lo largo de la obra de Leibniz podemos encontrar ciertos casos en los que la atención es atraída o capturada de forma involuntaria, como ocurre cuando despertamos a causa de una impresión sensible fuerte que atrapa nuestra atención (Leibniz, 1992: 119 [GP, V, 104]; véase también: Barth, 2011a: 41; y: Barth, 2016: 86). Con todo y esto, caracterizar la atención en términos de deseos o preferencias nos permite inferir que la atención, sea voluntaria o no, hace alusión a un cierto tipo de apetito o tendencia cognitiva que permite al sujeto advertir ciertas sensaciones (Barth, 2014: 347; véase también: Barth, 2016: 87).

La apercepción sensible, en ese sentido, mantiene una estructura compleja entre distinción, memoria y atención, la cual, sin embargo, no necesariamente va a acompañada de actos reflexivos. Esto último nos permite dos cosas: por un lado, salvaguardar la aparente inconsistencia que se presenta al hablar de la conciencia animal en Leibniz; por otro lado, nos permite distinguir entre la apercepción sensible y la conscientia, reservando la segunda para el caso específico de los espíritus.

\section{REFLEXIVIDAD Y CONSCIENTIA}

Mientras la apercepción sensible alude a un acto de primer orden que hace posible atender a algunas de nuestras percepciones sensibles más destacadas, como se aprecia en el pasaje del jabalí citado al inicio de esta investigación, el hannoveriano define la conscientia como "la reflexión sobre una acción, o la memoria de una acción que reconocemos como nuestra", lo cual "incluye la verdadera sustancia misma, o sea, al yo" (Leibniz, 2014c: 70 [Couturat, 495]). La conscientia, acorde con esta primera definición, consiste en un acto reflexivo o recuerdo (souvenir) inmediato que acompaña a la acción interna (Leibniz, 1992: 272 [GP, V, 220-221]; véase también: Jorgensen, 2011: 901ss), mediante el cual los espíritus alcanzan un conocimiento de lo que son y de lo que hacen, tal y como se puede apreciar en el \$34 de su Discours de métaphysique: "el alma inteligente, conociendo lo que es ella, y pudiendo decir ese $Y O$, que dice mucho, no sólo permanece y subsiste en sentido metafísico mucho más que las demás cosas, sino que 
además permanece la misma en sentido moral y constituye el mismo personaje. Es el recuerdo y el conocimiento de ese yo lo que la hace susceptible de castigo y recompensa" (Leibniz, 2010: 200 [AA, VI, 4B, 1584]).

Se trata, pues, de un acto reflexivo de segundo orden que no siempre está presente en los espíritus, ya que, si fuesen conscientes de todos sus actos, ello implicaría que, para cada reflexión habría otra reflexión, de modo que se daría una regresión al infinito que impediría progresar hacia nuevos pensamientos (Barth, 2011b:223; véase también: GP, VI, 600). La diferencia con la apercepción sensible radica en que, a pesar de que no todas las acciones de los espíritus estén acompañadas de actos reflexivos de la conscientia, los espíritus "siempre son conscientes de sus pensamientos de forma tal que, si alguien nos dirige, o si nosotros mismos nos dirigimos hacia nuestros pensamientos precedentes, sabríamos que los tuvimos" (AA, VI, 4B, 1471: la traducción es mía). Esto supone, en consecuencia, que nuestros pensamientos pueden 'ser dirigidos' a la conscientia, o bien por una causa externa, como ocurre cuando alguien nos interroga sobre lo que pensamos, o bien por una causa interna voluntaria que nos permite reparar en aquello que hay en nuestro interior (Barth, 2011b: 226-227). Ya sea externa o interna la causa, lo más relevante de esta afirmación se aprecia al enfatizar la relación entre la conscientia y la identidad personal, ya que con esto se afirma no sólo que la última no depende de una cierta continuidad psicológica, sino también que ésta puede ser evocada por otro, esto es, que el otro puede ayudarme a recordar quién soy, tal y como podemos observar en el siguiente pasaje de los Nouveaux essais sur l'entendement humain, el cual me permito citar en extenso:

Opino también que la conciencia o sentimiento del yo demuestra una identidad moral o personal. Y en esto se distinguen para mí la imposibilidad de cesar propia del alma de un animal de la inmortalidad del alma humana, una y otra conservan la identidad fisica y real, pero en lo que respecta al hombre, es conforme a las reglas de la divina providencia que el alma siga conservando la identidad moral y aparente a nosotros mismos, para constituir la misma persona, capaz por tanto de sentir castigos y recompensas. Parece ser que vos consideráis que esa identidad aparente podría conservarse aun cuando no hubiese identidad real. Creo que esto podría suceder, por el absoluto poder de Dios, pero de acuerdo con el orden de las cosas, la identidad aparente a la persona misma, que se siente igual a sí misma, supone la identidad real en cada próximo paso acompañado de reflexión o de sentimiento del yo: pues una percepción intima e inmediata no puede engañar naturalmente. Si el hombre tuviese que ser únicamente máquina, y tener a la vez conciencia de sí, habría que opinar como vos; pero me parece que ese caso no es posible, al menos naturalmente. Tampoco querría decir que la identidad personal e incluso el sí misino no se mantengan en nosotros y que yo ya no sea aquel yo que fui en la cuna, bajo pretexto de que ya no me 
acuerdo de nada de lo que hice entonces. Para encontrar la identidad moral por sí mismo basta que haya un vinculo medio de conciencia entre un estado próximo o incluso un poco alejado de otro, cuando algún salto o intervalo olvidado se mezcle en ello. Así, si una enfermedad supusiese una interrupción en el vínculo que establece la conciencia, de manera que no supiese cómo había podido llegar al estado presente, aun cuando me acuerde de las cosas más alejadas, el testimonio de los otros podría llenar el vacío de mi reminiscencia. Mediante este testimonio podría incluso ser castigado, si durante ese intervalo acabase de hacer algún mal a propósito, que poco después olvidé debido a esa enfermedad. Y si hubiese olvidado todas las cosas pasadas estaría obligado a dejarme enseñar de nuevo incluso mi nombre, y hasta leer y escribir, pero siempre podría aprender en los otros mi vida pasada durante el estado precedente, al igual que conserve mis derechos sin que fuera necesario partirme en dos personas, y nombrarme heredero de mí mismo. Todo eso es suficiente para mantener la identidad moral que constituye a la misma persona. (Leibniz, 1992: 270-271 [GP, V, 218-219])

Todo lo cual supone que la conscientia en Leibniz, además de constituir un tercer tipo de memoria, a saber, el recuerdo (souvenir), alude también a un tipo de atención que, de acuerdo con lo anterior, puede ser ocasionado tanto por el testimonio del otro, como por nuestra propia voluntad ${ }^{11}$. Me refiero, en particular, a aquella atención que prestamos a lo que hay en nosotros, incluyendo las ideas innatas (Kulstad, 1981: 33-34), en cuanto que, así como "somos, por así decirlo, innatos a nosotros mismos", asimismo "las ideas y las verdades nos son innatas, en tanto inclinaciones, disposiciones, hábitos o virtualidades naturales" (Leibniz, 1992: 40 [GP, $\mathrm{V}, 45])$. Esto último implica que los espíritus no sólo son capaces de prestar atención sobre el contenido de sus acciones, como ocurre con la apercepción sensible respecto a las percepciones sensibles, sino que también son capaces de adscribirse la acción en cuestión y, por tanto, de asumir responsabilidad por ella (Barth, 2011b: 229: véase también: Jorgensen, 2011: 902), en palabras del hannoveriano: "la reflexión no consiste en esto (el mero recuerdo de una acción); pues no sólo me represento mi acción, sino que también pienso que es mía o que soy yo quien hace o ha hecho esta acción" (GP, III, 299: la traducción y el paréntesis son míos; véase también: Leibniz, 2010: 332 [GP, VI, 612]).

11 Para estos efectos, conviene señalar que el hannoveriano define la voluntad como "la tendencia del ser inteligente" (Leibniz, 2014b: 63 [GP, VII, 297]), a partir de lo cual se sigue que los espíritus de orientar sus apetitos en función tanto de su conocimiento del mundo, como de su propio ser. de ahí que los espíritus no sólo gocen de una espontaneidad constitutiva, sino que también eleven sus actos al plano de la libertad, la cual define Leibniz como una "espontaneidad ligada a la inteligencia" (Leibniz, 2011a: 177 [GP, VII, 109]). 
Cuando se habla de la conscientia en Leibniz, a modo de conclusión de este apartado, se hace referencia a aquel conocimiento reflexivo de carácter introspectivo que permite al sujeto dos cosas: por un lado, adscribirse la causalidad de sus acciones y, en consecuencia, asumir responsabilidad por ellos; por otro lado, conservar su cualidad moral y, por tanto, su identidad personal. De ahí que, de acuerdo con la ontología monadológica del filósofo de Hannover, sólo los espíritus son capaces de deliberar, orientar o dirigir su estructura teleológica constitutiva y, por tanto, de concebirse a sí mismos como agentes morales, sin que por eso sean conscientes de todas sus acciones.

\section{CONCLUSIONES: LA TAXONOMÍA DE LAS MÓNADAS}

Al considerar la teoría leibniziana de la percepción que he presentado en el primer apartado de esta investigación y la reconstrucción que elaboro tanto su noción de apercepción sensible como su caracterización de la conscientia, en el segundo y tercer apartado respectivamente podemos articular su taxonomía de las mónadas en función de tres elementos: 2. el tipo de percepción; 2. la naturaleza de sus apetitos; y 3. el tipo de memoria que son capaces de alcanzar acorde a su grado de perfección. Todas las mónadas, acorde a lo mencionado en los apartados anteriores, poseen al menos las primeras dos, mientras que la conciencia sólo se presenta en las almas y los espíritus, tal y como se presenta en el siguiente cuadro:

\begin{tabular}{|l|l|l|l|l|}
\hline & Mónadas & Almas & Espíritus \\
\cline { 3 - 4 } simples & Inferiores & Complejas & Pensamientos \\
\hline Percepción & $\begin{array}{l}\text { Pequeñas } \\
\text { percepciones }\end{array}$ & $\begin{array}{l}\text { Percepcio- } \\
\text { nes sensibles }\end{array}$ & Sensación & $\begin{array}{l}\text { e ideas inna- } \\
\text { tas }\end{array}$ \\
\hline Apetición & $\begin{array}{l}\text { Apetitos cie- } \\
\text { gos o pasivos }\end{array}$ & $\begin{array}{l}\text { Apetitos } \\
\text { cognitivos } \\
\text { sensibles }\end{array}$ & Atención & Voluntad \\
\hline Memoria & $\begin{array}{l}\text { Memoria vir- } \\
\text { tual u onto- } \\
\text { lógica }\end{array}$ & $\begin{array}{l}\text { Memoria vir- } \\
\text { tual u onto- } \\
\text { lógica }\end{array}$ & $\begin{array}{l}\text { Reminiscen- } \\
\text { cia }\end{array}$ & $\begin{array}{l}\text { Recuerdo } \\
\text { (souvenir) }\end{array}$ \\
\hline
\end{tabular}

Cuadro: Taxonomía de las mónadas. Elaboración propia.

Las mónadas simples, en primer lugar, constituyen unidades sustanciales cuyas percepciones y apetitos carecen por completo de distinción, de modo que sus los trazos de las primeras, a pesar de ser almacenadas en la memoria, no poseen la fuerza suficiente para ser repetidas. Se trata, en 
principio, de mónadas radicalmente pasivas. En segundo lugar, se encuentran las almas, cuyas percepciones y apetitos se elevan al grado de la sensación, sin por eso poseer la fuerza suficiente para producir una disposición habitual que sea susceptible de ser rememorada, lo cual nos permite distinguir dos tipos de almas. Al primer tipo de almas se les caracteriza por poseer sensaciones que, sin embargo, carecen de la fuerza suficiente como para generar de golpe ese eco o disposición habitual en el alma; mientras que un segundo tipo de almas se concibe en aquellos animales cuyos órganos sensoriales poseen una estructura compleja, capaz de reunir varias impresiones sensibles y generar, en consecuencia, una impresión más fuerte. Esta última, a su vez, se corresponde con una representación mental cuyo relieve es suficiente para originar una disposición habitual en el alma, la cual es susceptible de ser rememorada en virtud de un apetito cognitivo que nos permite reparar en la sensación, esto es, la atención.

Dada la presencia de este último tipo de apetito, este segundo tipo de almas poseen percepciones que les permite tener reminiscencia y, por tanto, cierto tipo de consecuciones que emulan a la razón, sin por eso establecer conexiones necesarias entre los fenómenos apercibidos. Esto implica que sólo este segundo tipo de vivientes poseen apercepción sensible o conciencia fenoménica, más no conscientia, para lo cual es necesario que sean susceptibles de poseer actos reflexivos. Este es el caso, por último, de los espíritus, cuyas percepciones no se limitan al ámbito de lo fenoménico o sensible, de forma que sus actos mentales se elevan al ámbito de la razón, esto es, de la intelección y de las ideas. Sólo los espíritus, en sentido estricto, gozan de actos reflexivos, lo cual les permite autoadscribirse la causalidad de sus acciones y, por tanto, concebirse como agentes morales.

\section{REFERENCIAS}

Andreu, A. (2015). Unidad infinito novella. En G. W. Leibniz, Methodus Vitae (vol. II, Individuo o mónada, pp. XIII-LIV). Madrid: Plaza y Valdés.

Barth, C. (2011a). Apperception in the New Essays Concerning Human Understanding. A Critique of the Reflective Account and a Sketch of an Alternative Proposal. En H. Breger (ed.), Natur und Subjekt: IX Internationaler Leibniz-Kongress unter der Schirmberrschaft des Bundespräsidenten, Hannover, 26. September bis 1. Oktober 2011; Vortrage (vol. 1, pp. 37-43), Hannover.

Barth, C. (2011b). Leibnitian Conscientia and its Cartesian Roots. Studia Leibnitiana, 43(2), 216-236.

Barth, C. (2014). Leibniz on Phenomenal Consciousness. Vivarium, (52), 333357.

Barth, C. (2016). Leibniz's Conception of Sensation. En A. Pelletier (ed.), Leibniz's Experimental Pbilosophy (pp. 64-70), Studia Leibnitiana, 46, Stuttgard: Franz Steiner Verlag. 
Descartes, R. (1982-91): Oeuvres de Descartes (C. Adam and P. Tannery, eds., 12 vols.). Paris: J. Vrin.

Gennaro, R. J. (1999). Leibniz on Consciousness and Self-Consciousness. En R. J. Gennaro and C. Huenemann (eds.), New Essays on the Rationalists (pp. 353371). Nueva York: Oxford University Press.

Herrera, A. (1993). Leibniz y su visión ontológica de la percepción. En L. Benítez y J. A. Robles (comp.), Percepción: colores (pp. 91-100). Ciudad de México: UNAM.

Jorgensen, L. (2009). The Principle of Continuity and Leibniz's Theory of Consciousness. Journal of the History of Philosophy, 47(2), 223-248.

Jorgensen, L. (2011). Leibniz on Memory and Consciousness. British Journal for the History of Philosophy, 19(5), 887-916.

Kulstad, M. (1981). Leibniz, Animals, and Apperception. Studia Leibnitiana, 13(1), $25-60$.

Leibniz, G. W. (1923). [AA]. Sämtliche Schriften und Briefe. Darmstadt (1923 y ss.), Leipzig (1938 y ss.), Berlín (1950 y ss.): Deutschen Akademie der Wissenschaften zu Berlin.

Leibniz, G. W. (1948). [Grua]. Textes inédits d'après les manuscrites de la bibliothèque proviciale de Hannovre. (Publiés et annotés par G. Grua). Paris: Presses Universitaires de France.

Leibniz, G. W. (1954). [Robinet I]. Principes de la nature et de la grâce fondés en raison. Principes de la philosophie ou monadologie. (Publiés intégralement d'après des lettres inédites par A. Robinet). París: Presses Universitaires de France.

Leibniz, G. W. (1961). [Couturat]. Opuscules et fragmentes inédits de Leibniæ. Extraits des manuscrits de la Bibliothèque royale de Hanovre. (par L. Couturat): Hildesheim.

Leibniz, G. W. (1965). [GP]. Die Philosophischen Schriften. (herausgegeben von C.I. Gerhardt): Hildesheim.

Leibniz, G. W. (1992). Nuevos ensayos sobre el entendimiento bumano. (J. Echeverría, Trad.). Madrid: Alianza.

Leibniz, G. W. (1997). [Finster]. Der Briefwechsel mit Antoine Arnauld. (R. de Finster, F. Meiner). Verlag, Hamburg.

Leibniz, G. W. (2007). G. W. Leibniz. Obras filosóficas y cientificas. 14. Correspondencia I. (J. A. Nicolás, M. Ramón Cubells, eds.). Granada: Comares.

Leibniz, G. W. (2010). G.W. Leibniz: Obras filosóficas y científicas. 2. Metafísica. (A. L. González, ed.). Granada: Comares.

Leibniz, G. W. (2011a). Gottfried Wilhelm Leibniz. (J. Echeverría, ed.). Madrid: Gredos.

Leibniz, G. W. (2011b). G. W. Leibniz: Obras filosóficas y científicas. 16 B. Correspondencia III. (B. Orio de Miguel, ed.). Granada: Comares.

Leibniz, G. W. (2012). G. W. Leibniz. Obras filosóficas y cientificas. 10. Ensayos de Teodicea. (T. Guillén Vera, ed.). Granada: Comares.

Leibniz, G. W. (2014a). Methodus Vitae (Escritos de Leibniz). Vol. I: Naturaleza o fuerza. (A. Andreu, Trad.). Madrid: Plaza y Valdés.

Leibniz, G. W. (2014b). Methodus Vitae (Escritos de Leibniz). Vol. II: Individuo o mónada. (A. Andreu, Trad.). Madrid: Plaza y Valdés. 
Leibniz, G. W. (2014c). Methodus Vitae (Escritos de Leibniz). Vol. III: Ética o política (A. Andreu, Trad.). Madrid: Plaza y Valdés.

McRae, R. (1978). Leibniz: Perception, Apperception and Thought. Toronto: Toronto University Press.

Rescher, N. (1979). Leibniz: An Introduction to his Philosophy. Oxford: Basil Blackwell.

Rovira, R. (2006). Léxico fundamental de la Metafísica de Leibniz. Madrid: Trotta.

Rutherford, D. (2005). Leibniz on Spontaneity. En D. Rutherford y J. A. Cover (eds.), Leibniz: Nature and Freedom (pp. 156-180). Nueva York: Oxford University Press.

Simmons, A. (2011). Changing the Cartesian Mind: Leibniz on Sensation, Representation and Consciousness. The Philosophical Review, 110(1), 31-75.

Sumario: Introducción; 1. Percepción y apetición como principios internos del cambio; 2. Apercepción sensible o conciencia fenoménica; 3. Reflexividad y conscientia; Conclusiones: la taxonomía de las mónadas; Referencias. 